\title{
Some New Identities for the Generalized Fibonacci Polynomials by the $Q(x)$ Matrix
}

\author{
Chung-Chuan Chen ${ }^{1}$ \& Lin-Ling Huang ${ }^{1}$ \\ ${ }^{1}$ Department of Mathematics Education, National Taichung University of Education, Taiwan \\ Correspondence: Chung-Chuan Chen, Department of Mathematics Education, National Taichung University of Educa- \\ tion, Taiwan. E-mail: chungchuan@mail.ntcu.edu.tw
}

Received: January 26, 2021 Accepted: March 3, 2021 Online Published: March 7, 2021

doi:10.5539/jmr.v13n2p21 URL: https://doi.org/10.5539/jmr.v13n2p21

\begin{abstract}
We obtain some new identities for the generalized Fibonacci polynomial by a new approach, namely, the $Q(x)$ matrix. These identities including the Cassini type identity and Honsberger type formula can be applied to some polynomial sequences such as Fibonacci polynomials, Lucas polynomials, Pell polynomials, Pell-Lucas polynomials and so on, which generalize the previous results in references.
\end{abstract}

Keywords: generalized Fibonacci polynomial, $Q(x)$ matrix, Cassini type identity, Honsberger type formula

\section{Introduction}

A second order polynomial sequence $F_{n}(x)$ is said to be the Fibonacci polynomial if for $n \geq 2$ and $x \in \mathbb{R}$,

$$
F_{n}(x)=x F_{n-1}(x)+F_{n-2}(x)
$$

with $F_{0}(x)=0$ and $F_{1}(x)=1$. The Fibonacci polynomial and other polynomials attracted a lot of attention over the last several decades (see, for instance, Falcón \& Plaza, 2007, 2009; Gould, 1981; Horadam, 1979; Horadam \& Mahon, 1985; Wu \& Zhang, 2013). Recently, the generalized Fibonacci polynomial is introduced and studied intensely by many authors (André-Jeannin, 1994, 1995; Flórez, Higuita \& Mukherjee, 2018; Flórez, McAnally \& Mukherjee, 2018), which is a generalization of the Fibonacci polynomial. Indeed, a polynomial sequence $G_{n}(x)$ in (Flórez, Higuita \& Mukherjee, 2018; Flórez, McAnally \& Mukherjee, 2018) is called the generalized Fibonacci polynomial if for $n \geq 2$,

$$
G_{n}(x)=c(x) G_{n-1}(x)+d(x) G_{n-2}(x)
$$

with $G_{0}(x)$ and $G_{1}(x)$, where $c(x)$ and $d(x)$ are fixed non-zero polynomials in $\mathbb{Q}[x]$. It should be noted that there is no unique generalization of Fibonacci polynomials. Following the similar definitions in (Flórez, McAnally \& Mukherjee, 2018 ), in this note, $\mathcal{F}_{n}(x)$ is said to be the Fibonacci type polynomial if for $n \geq 2$,

$$
\mathcal{F}_{0}(x)=0, \mathcal{F}_{1}(x)=a \text { and } \mathcal{F}_{n}(x)=c(x) \mathcal{F}_{n-1}(x)+d(x) \mathcal{F}_{n-2}(x)
$$

where $a \in \mathbb{R} \backslash\{0\}$. If for $n \geq 2$,

$$
\mathcal{L}_{0}(x)=q, \mathcal{L}_{1}(x)=b(x) \text { and } \mathcal{L}_{n}(x)=c(x) \mathcal{L}_{n-1}(x)+d(x) \mathcal{L}_{n-2}(x),
$$

then the polynomial sequence $\mathcal{L}_{n}(x)$ is called the Lucas type polynomial, where $q \in \mathbb{R} \backslash\{0\}$ and $b(x)$ is a fixed non-zero polynomial in $\mathbb{Q}[x]$. Naturally, both $\mathcal{F}_{n}(x)$ and $\mathcal{L}_{n}(x)$ are the generalized Fibonacci polynomials. We note that if we assume $\mathcal{F}_{1}(x)=a=1$, then $\mathcal{F}_{n}(x)$ is the Fibonacci type polynomial given in (Flórez, McAnally \& Mukherjee, 2018). In addition, the definition of $\mathcal{L}_{n}(x)$ is the same with that of Flórez et al (Flórez, McAnally \& Mukherjee, 2018) if $|q|=1$ or 2 , and $c(x)=\frac{2}{q} b(x)$. In other words, our definitions of $\mathcal{F}_{n}(x)$ and $\mathcal{L}_{n}(x)$ are generalizations of those in (Flórez, McAnally $\&$ Mukherjee, 2018).

Our goal is to give some new identities for $\mathcal{F}_{n}(x)$ and $\mathcal{L}_{n}(x)$ by applying a new approach, namely the $Q(x)$ matrix, rather than using the Binet formulas. We note that in some references, the authors obtain identities for polynomial sequences by using the Binet formulas. Here, we provide a new way to deduce some new identities for $\mathcal{F}_{n}(x)$ and $\mathcal{L}_{n}(x)$. Besides, these results can be applied to some familiar polynomial sequences. Indeed, the polynomial sequences in the upper part of Table 1 below are the Fibonacci type polynomials. On the other hand, those in the lower part of Table 1 are the Lucas type polynomials. Table 1 is the rearrangement of Table 1 (Flórez, McAnally \& Mukherjee, 2018). 
Table 1. Some examples of the generalized Fibonacci polynomials

\begin{tabular}{lccc}
\hline Polynomial & Initial value & Initial value & Recursive Formula \\
& $G_{0}(x)$ & $G_{1}(x)$ & $G_{n}(x)=c(x) G_{n-1}(x)+d(x) G_{n-2}(x)$ \\
\hline \hline Fibonacci & 0 & 1 & $F_{n}(x)=x F_{n-1}(x)+F_{n-2}(x)$ \\
Pell & 0 & 1 & $P_{n}(x)=2 x P_{n-1}(x)+P_{n-2}(x)$ \\
Fermat & 0 & 1 & $\Phi_{n}(x)=3 x \Phi_{n-1}(x)-2 \Phi_{n-2}(x)$ \\
Chebyshev second kind & 0 & 1 & $U_{n}(x)=2 x U_{n-1}(x)-U_{n-2}(x)$ \\
Jacobsthal & 0 & 1 & $J_{n}(x)=J_{n-1}(x)+2 x J_{n-2}(x)$ \\
Morgan-Voyce & 0 & 1 & $B_{n}(x)=(x+2) B_{n-1}(x)-B_{n-2}(x)$ \\
Vieta & 0 & 1 & $V_{n}(x)=x V_{n-1}(x)-V_{n-2}(x)$ \\
\hline Lucas & 2 & $x$ & $L_{n}(x)=x L_{n-1}(x)+L_{n-2}(x)$ \\
Pell-Lucas & 2 & $2 x$ & $D_{n}(x)=2 x D_{n-1}(x)+D_{n-2}(x)$ \\
Pell-Lucas-prime & 1 & $x$ & $D_{n}^{\prime}(x)=2 x D_{n-1}^{\prime}(x)+D_{n-2}^{\prime}(x)$ \\
Fermat-Lucas & 2 & $3 x$ & $\vartheta_{n}(x)=3 x \vartheta_{n-1}(x)-2 \vartheta_{n-2}(x)$ \\
Chebyshev first kind & 1 & $x$ & $T_{n}(x)=2 x T_{n-1}(x)-T_{n-2}(x)$ \\
Jacobsthal-Lucas & 1 & 1 & $\Lambda_{n}(x)=\Lambda_{n-1}(x)+2 x \Lambda_{n-2}(x)$ \\
Morgan-Voyce & 2 & $x+2$ & $C_{n}(x)=(x+2) C_{n-1}(x)-C_{n-2}(x)$ \\
Vieta-Lucas & 2 & $x$ & $v_{n}(x)=x v_{n-1}(x)-v_{n-2}(x)$ \\
\hline
\end{tabular}

\section{Fibonacci Type Polynomials}

In this section, we will provide and prove some identities for the Fibonacci type polynomial $\mathcal{F}_{n}(x)$ by applying the Fibonacci type $Q(x)$ matrix. The original Fibonacci $Q$ matrix was introduced by Charles H. King in his master thesis (cf. Koshy, 2001), and given by

$$
Q=\left(\begin{array}{ll}
1 & 1 \\
1 & 0
\end{array}\right) .
$$

The Fibonacci $Q$ matrix is connected to the Fibonacci sequence $F_{n}$, which is defined as below

$$
F_{0}=1, F_{1}=1 \text { and } F_{n}=F_{n-1}+F_{n-2} \text { for } n \geq 2 .
$$

Indeed, it is noted in (Gould, 1981) that

$$
Q^{n}=\left(\begin{array}{ll}
1 & 1 \\
1 & 0
\end{array}\right)^{n}=\left(\begin{array}{cc}
F_{n} & F_{n-1} \\
F_{n-1} & F_{n-2}
\end{array}\right) .
$$

Using this relation above, some familiar identities can be obtained. For instance,

$$
\operatorname{det}\left(\begin{array}{cc}
F_{n+1} & F_{n} \\
F_{n} & F_{n-1}
\end{array}\right)=\left(\operatorname{det}\left(\begin{array}{ll}
1 & 1 \\
1 & 0
\end{array}\right)\right)^{n}
$$

implies the Cassini identity

$$
F_{n+1} F_{n-1}-F_{n}^{2}=(-1)^{n} .
$$

Also, using this equality $Q^{n+m}=Q^{n} Q^{m}$, one can deduce the Honsberger formula.

In the following, we will apply some similar idea of $Q$ matrix from the numerical cases (Lin, 2012) to the Fibonacci type polynomials $\mathcal{F}_{n}(x)$. For $n \geq 2$ and $x \in \mathbb{R}$, the Fibonacci type polynomial $\mathcal{F}_{n}(x)$ is defined by

$$
\mathcal{F}_{0}(x)=0, \mathcal{F}_{1}(x)=a \text { and } \mathcal{F}_{n}(x)=c(x) \mathcal{F}_{n-1}(x)+d(x) \mathcal{F}_{n-2}(x)
$$

where $a \in \mathbb{R} \backslash\{0\}$. Then

$$
\left(\begin{array}{c}
\mathcal{F}_{n+2}(x) \\
\mathcal{F}_{n+1}(x)
\end{array}\right)=\left(\begin{array}{cc}
c(x) & d(x) \\
1 & 0
\end{array}\right)\left(\begin{array}{c}
\mathcal{F}_{n+1}(x) \\
\mathcal{F}_{n}(x)
\end{array}\right)
$$

Here we define the Fibonacci type $Q(x)$ matrix by

$$
Q(x)=\left(\begin{array}{cc}
c(x) & d(x) \\
1 & 0
\end{array}\right) .
$$

We note that if $\mathcal{F}_{n}(x)=P_{n}(x)$ is the Pell polynomial as defined in Table 1, then

$$
Q(x)=\left(\begin{array}{cc}
2 x & 1 \\
1 & 0
\end{array}\right)
$$


which appeared in (Horadam \& Mahon, 1985). In addition, we observe that

$$
\left(\begin{array}{c}
\mathcal{F}_{n+2}(x) \\
\mathcal{F}_{n+1}(x)
\end{array}\right)=\left(\begin{array}{cc}
c(x) & d(x) \\
1 & 0
\end{array}\right)^{n}\left(\begin{array}{c}
\mathcal{F}_{2}(x) \\
\mathcal{F}_{1}(x)
\end{array}\right)=\left(\begin{array}{cc}
c(x) & d(x) \\
1 & 0
\end{array}\right)^{n}\left(\begin{array}{c}
a c(x) \\
a
\end{array}\right) .
$$

On the other hand,

$$
\left(\begin{array}{l}
\mathcal{F}_{n+2}(x) \\
\mathcal{F}_{n+1}(x)
\end{array}\right)=\left(\begin{array}{c}
c(x) \mathcal{F}_{n+1}(x)+d(x) \mathcal{F}_{n}(x) \\
c(x) \mathcal{F}_{n}(x)+d(x) \mathcal{F}_{n-1}(x)
\end{array}\right)=\left(\begin{array}{cc}
\frac{1}{a} \mathcal{F}_{n+1}(x) & \frac{d(x)}{a} \mathcal{F}_{n}(x) \\
\frac{1}{a} \mathcal{F}_{n}(x) & \frac{d(x)}{a} \mathcal{F}_{n-1}(x)
\end{array}\right)\left(\begin{array}{c}
a c(x) \\
a
\end{array}\right) .
$$

Hence the below result follows.

Theorem 2.1 Let $\mathcal{F}_{n}(x)$ be the Fibonacci type polynomial as defined in Eq. (1). Then for each $n \in \mathbb{N}$,

$$
\left(\begin{array}{cc}
\frac{1}{a} \mathcal{F}_{n+1}(x) & \frac{d(x)}{a} \mathcal{F}_{n}(x) \\
\frac{1}{a} \mathcal{F}_{n}(x) & \frac{d(x)}{a} \mathcal{F}_{n-1}(x)
\end{array}\right)=\left(\begin{array}{cc}
c(x) & d(x) \\
1 & 0
\end{array}\right)^{n}=Q^{n}(x) .
$$

Proof. Let $n=1$. Then

$$
\left(\begin{array}{ll}
\frac{1}{a} \mathcal{F}_{2}(x) & \frac{d(x)}{a} \mathcal{F}_{1}(x) \\
\frac{1}{a} \mathcal{F}_{1}(x) & \frac{d(x)}{a} \mathcal{F}_{0}(x)
\end{array}\right)=\left(\begin{array}{cc}
c(x) & d(x) \\
1 & 0
\end{array}\right)
$$

Assume the equality holds for $n=k$. Then we have

$$
\left(\begin{array}{cc}
\frac{1}{a} \mathcal{F}_{k+1}(x) & \frac{d(x)}{a} \mathcal{F}_{k}(x) \\
\frac{1}{a} \mathcal{F}_{k}(x) & \frac{d(x)}{a} \mathcal{F}_{k-1}(x)
\end{array}\right)=\left(\begin{array}{cc}
c(x) & d(x) \\
1 & 0
\end{array}\right)^{k}
$$

If $n=k+1$, then

$$
\left(\begin{array}{ll}
\frac{1}{a} \mathcal{F}_{k+2}(x) & \frac{d(x)}{a} \mathcal{F}_{k+1}(x) \\
\frac{1}{a} \mathcal{F}_{k+1}(x) & \frac{d(x)}{a} \mathcal{F}_{k}(x)
\end{array}\right)=\left(\begin{array}{cc}
c(x) & d(x) \\
1 & 0
\end{array}\right)\left(\begin{array}{cc}
\frac{1}{a} \mathcal{F}_{k+1}(x) & \frac{d(x)}{a} \mathcal{F}_{k}(x) \\
\frac{1}{a} \mathcal{F}_{k}(x) & \frac{d(x)}{a} \mathcal{F}_{k-1}(x)
\end{array}\right)=\left(\begin{array}{cc}
c(x) & d(x) \\
1 & 0
\end{array}\right)^{k+1}
$$

By induction, the result follows.

The Cassini type identity of the Fibonacci type polynomial $\mathcal{F}_{n}(x)$ can be obtained below by Theorem 2.1.

Corollary 2.2 Let $\mathcal{F}_{n}(x)$ be the Fibonacci type polynomial. Then for each $n \in \mathbb{N}$,

$$
\mathcal{F}_{n}^{2}(x)-\mathcal{F}_{n+1}(x) \mathcal{F}_{n-1}(x)=a^{2}(-d(x))^{n-1} .
$$

Proof. By Theorem 2.1,

$$
\operatorname{det}\left(\begin{array}{cc}
\frac{1}{\mathcal{F}_{n+1}(x)} & \frac{d(x)}{a} \mathcal{F}_{n}(x) \\
\frac{1}{a} \mathcal{F}_{n}(x) & \frac{d(x)}{a} \mathcal{F}_{n-1}(x)
\end{array}\right)=\left(\operatorname{det}\left(\begin{array}{cc}
c(x) & d(x) \\
1 & 0
\end{array}\right)\right)^{n}
$$

Hence

$$
\mathcal{F}_{n}^{2}(x)-\mathcal{F}_{n+1}(x) F_{n-1}(x)=a^{2}(-d(x))^{n-1} .
$$

Example 2.3 Let $a=1, c(x)=x, d(x)=1$ in Eq. (1). Then $\mathcal{F}_{n}(x)$ is the classical Fibonacci polynomial $F_{n}(x)$. By Corollary 2.2, we recover the Cassini identity in (Falcón $\mathcal{E}$ Plaza, 2009),

$$
F_{n+1}(x) F_{n-1}(x)-F_{n}^{2}(x)=(-1)^{n} .
$$

Example 2.4 Let $\mathcal{F}_{n}(x)$ be the Pell polynomial $P_{n}(x)$ as defined in Table 1. By Corollary 2.2,

$$
P_{n+1}(x) P_{n-1}(x)-P_{n}^{2}(x)=(-1)^{n}
$$

which is the identity (2.5) in (Horadam $\mathcal{E}$ Mahon, 1985). 
Example 2.5 Let $a=1, c(x)=1, d(x)=2 x$ in Eq. (1). Then $\mathcal{F}_{n}(x)=J_{n}(x)$ is the Jacobsthal polynomial as defined in Table 1. By Corollary 2.2, one can obtain the Cassini identity for the Jacobsthal polynomial below

$$
J_{n}^{2}(x)-J_{n+1}(x) J_{n-1}(x)=(-2 x)^{n-1} .
$$

By Corollary 2.2, we have the result below.

Corollary 2.6 Let $\mathcal{F}_{n}(x)$ be the Fibonacci type polynomial. Then for each $n \in \mathbb{N}$,

$$
\mathcal{F}_{n}^{2}(x)-c(x) \mathcal{F}_{n}(x) \mathcal{F}_{n-1}(x)-d(x) \mathcal{F}_{n-1}^{2}(x)=a^{2}(-d(x))^{n-1} .
$$

Proof. By

$$
\mathcal{F}_{n}^{2}(x)-\mathcal{F}_{n+1}(x) \mathcal{F}_{n-1}(x)=a^{2}(-d(x))^{n-1} .
$$

and

$$
\mathcal{F}_{n+1}(x)=c(x) \mathcal{F}_{n}(x)+d(x) \mathcal{F}_{n-1}(x)
$$

we have

$$
\begin{aligned}
a^{2}(-d(x))^{n-1} & =\mathcal{F}_{n}^{2}(x)-\left(c(x) \mathcal{F}_{n}(x)+d(x) \mathcal{F}_{n-1}(x)\right) \mathcal{F}_{n-1}(x) \\
& =\mathcal{F}_{n}^{2}(x)-c(x) \mathcal{F}_{n}(x) \mathcal{F}_{n-1}(x)-d(x) \mathcal{F}_{n-1}^{2}(x)
\end{aligned}
$$

By applying $Q^{n+m}(x)=Q^{n}(x) Q^{m}(x)$, we give the Honsberger type formula for the Fibonacci type polynomials $\mathcal{F}_{n}(x)$ below.

Corollary 2.7 Let $\mathcal{F}_{n}(x)$ be the Fibonacci type polynomial. Then for each $n, m \in \mathbb{N}$,

$$
a \mathcal{F}_{n+m}(x)=\mathcal{F}_{n}(x) \mathcal{F}_{m+1}(x)+d(x) \mathcal{F}_{n-1}(x) \mathcal{F}_{m}(x) .
$$

Proof. By

$$
\left(\begin{array}{cc}
c(x) & d(x) \\
1 & 0
\end{array}\right)^{n+m}=\left(\begin{array}{cc}
c(x) & d(x) \\
1 & 0
\end{array}\right)^{n}\left(\begin{array}{cc}
c(x) & d(x) \\
1 & 0
\end{array}\right)^{m}
$$

we have

$$
\left(\begin{array}{cc}
\frac{1}{a} \mathcal{F}_{n+m+1}(x) & \frac{d(x)}{a} \mathcal{F}_{n+m}(x) \\
\frac{1}{a} \mathcal{F}_{n+m}(x) & \frac{d(x)}{a} \mathcal{F}_{n+m-1}(x)
\end{array}\right)=\left(\begin{array}{cc}
\frac{1}{a} \mathcal{F}_{n+1}(x) & \frac{d(x)}{a} \mathcal{F}_{n}(x) \\
\frac{1}{a} \mathcal{F}_{n}(x) & \frac{d(x)}{a} \mathcal{F}_{n-1}(x)
\end{array}\right)\left(\begin{array}{cc}
\frac{1}{a} \mathcal{F}_{m+1}(x) & \frac{d(x)}{a} \mathcal{F}_{m}(x) \\
\frac{1}{a} \mathcal{F}_{m}(x) & \frac{d(x)}{a} \mathcal{F}_{m-1}(x)
\end{array}\right) .
$$

Hence by the $(2,1)$ entry of the first matrix in the equality above,

$$
a \mathcal{F}_{n+m}(x)=\mathcal{F}_{n}(x) \mathcal{F}_{m+1}(x)+d(x) \mathcal{F}_{n-1}(x) \mathcal{F}_{m}(x) .
$$

\section{Remark 2.8}

(i) Let $a=1$ in Corollary 2.7. Then Corollary 2.7 is the same with the first result of Proposition 1 (Flórez, McAnally $\mathcal{E}$ Mukherjee, 2018), and a generalization of Proposition 5 (Falcón $\mathcal{E}$ Plaza, 2009).

(ii) If $m=n-1$ in the above corollary, then for each $n \in \mathbb{N}$,

$$
a \mathcal{F}_{2 n-1}(x)=\mathcal{F}_{n}^{2}(x)+d(x) \mathcal{F}_{n-1}^{2}(x)
$$

which generalizes the numerical case of Fibonacci sequences. 
Example 2.9 Let $a=1, c(x)=x, d(x)=1$ in Eq. (1). Then $\mathcal{F}_{n}(x)=F_{n}(x)$ is the Fibonacci polynomial as defined in Table 1. By Corollary 2.7, we recover the Honsberger formula in Proposition 5 (Falcón E Plaza, 2009),

$$
F_{n+m}(x)=F_{n}(x) F_{m+1}(x)+F_{n-1}(x) F_{m}(x) .
$$

Example 2.10 Let $a=1, c(x)=2 x, d(x)=1$ in Eq. (1). Then $\mathcal{F}_{n}(x)$ is the Pell polynomial $P_{n}(x)$. By Corollary 2.7, we have

$$
P_{n+m}(x)=P_{n}(x) P_{m+1}(x)+P_{n-1}(x) P_{m}(x)
$$

which is the equality (3.14) in (Horadam $\mathcal{E}$ Mahon, 1985).

Using $Q^{n-m}(x)=Q^{n}(x) Q^{-m}(x)$ for $n \geq m$, we next will prove the d'Ocagne type identity for $\mathcal{F}_{n}(x)$. Here we need to assume $d(x) \neq 0$ for each $x \in \mathbb{R}$ so that $Q(x)$ is invertible. Moreover, note that

$$
Q^{-m}(x)=\left(\begin{array}{cc}
\frac{1}{a} \mathcal{F}_{m+1}(x) & \frac{d(x)}{a} \mathcal{F}_{m}(x) \\
\frac{1}{a} \mathcal{F}_{m}(x) & \frac{d(x)}{a} \mathcal{F}_{m-1}(x)
\end{array}\right)^{-1}=\frac{1}{(-d(x))^{m}}\left(\begin{array}{cc}
\frac{d(x)}{a} \mathcal{F}_{m-1}(x) & -\frac{d(x)}{a} \mathcal{F}_{m}(x) \\
-\frac{1}{a} \mathcal{F}_{m}(x) & \frac{1}{a} \stackrel{\mathcal{F}}{m+1}_{m}(x)
\end{array}\right)
$$

by Theorem 2.1 and Corollary 2.2.

Corollary 2.11 Let $\mathcal{F}_{n}(x)$ be the Fibonacci type polynomial, and let $d(x) \neq 0$ for each $x \in \mathbb{R}$. Then for $n, m \in \mathbb{N}$ with $n \geq m$,

$$
a(-d(x))^{m} \mathcal{F}_{n-m}(x)=\mathcal{F}_{n}(x) \mathcal{F}_{m+1}(x)-\mathcal{F}_{n+1}(x) \mathcal{F}_{m}(x) .
$$

Proof. By $Q^{n-m}(x)=Q^{n}(x) Q^{-m}(x)$, we have

$$
\begin{aligned}
& \left(\begin{array}{cc}
\frac{1}{a} \mathcal{F}_{n-m+1}(x) & \frac{d(x)}{a} \mathcal{F}_{n-m}(x) \\
\frac{1}{a} \mathcal{F}_{n-m}(x) & \frac{d(x)}{a} \mathcal{F}_{n-m-1}(x)
\end{array}\right) \\
= & \left(\begin{array}{cc}
\frac{1}{a} \mathcal{F}_{n+1}(x) & \frac{d(x)}{a} \mathcal{F}_{n}(x) \\
\frac{1}{a} \mathcal{F}_{n}(x) & \frac{d(x)}{a} \mathcal{F}_{n-1}(x)
\end{array}\right) \frac{1}{(-d(x))^{m}}\left(\begin{array}{cc}
\frac{d(x)}{a} \mathcal{F}_{m-1}(x) & -\frac{d(x)}{a} \mathcal{F}_{m}(x) \\
-\frac{1}{a} \mathcal{F}_{m}(x) & \frac{1}{a} \mathcal{F}_{m+1}(x)
\end{array}\right) .
\end{aligned}
$$

Hence considering the $(1,2)$ entry of the first matrix in the equality above,

$$
a(-d(x))^{m} \mathcal{F}_{n-m}(x)=\mathcal{F}_{n}(x) \mathcal{F}_{m+1}(x)-\mathcal{F}_{n+1}(x) \mathcal{F}_{m}(x) .
$$

Example 2.12 $\operatorname{Let} \mathcal{F}_{n}(x)$ be the Fibonacci polynomial $F_{n}(x)$ as in Table 1. By Corollary 2.11,

$$
(-1)^{m} F_{n-m}(x)=F_{n}(x) F_{m+1}(x)-F_{n+1}(x) F_{m}(x)
$$

which is the d'Ocagne identity in Corollary 8 (Falcón $\mathcal{E}$ Plaza, 2009), and the identity (47) of Proposition 3 (Flórez, McAnally \& Mukherjee, 2018).

We note that $Q(x)=\left(\begin{array}{cc}c(x) & d(x) \\ 1 & 0\end{array}\right)$ satisfies $Q^{2}(x)=c(x) Q(x)+d(x) I$ where $I=\left(\begin{array}{ll}1 & 0 \\ 0 & 1\end{array}\right)$. Using this equality, one can obtain the following expression of $\mathcal{F}_{n}(x)$.

Theorem 2.13 Let $\mathcal{F}_{n}(x)$ be the Fibonacci type polynomial. Then for each $n, p \in \mathbb{N}$,

$$
\mathcal{F}_{2 n+p}(x)=\sum_{j=0}^{n}\left(\begin{array}{c}
n \\
j
\end{array}\right) c^{j}(x) d^{n-j}(x) \mathcal{F}_{j+p}(x)
$$


Proof. Consider

$$
\begin{aligned}
& \left(\begin{array}{cc}
\frac{1}{a} \mathcal{F}_{2 n+p+1}(x) & \frac{d(x)}{a} \mathcal{F}_{2 n+p}(x) \\
\frac{1}{a} \mathcal{F}_{2 n+p}(x) & \frac{d(x)}{a} \mathcal{F}_{2 n+p-1}(x)
\end{array}\right) \\
= & Q^{2 n+p}(x) \\
= & Q^{p}(x)\left(Q^{2}(x)\right)^{n} \\
= & Q^{p}(x)(c(x) Q(x)+d(x) I)^{n} \\
= & Q^{p}(x)\left(\sum_{j=0}^{n}\left(\begin{array}{c}
n \\
j
\end{array}\right) c^{j}(x) d^{n-j}(x) Q^{j}(x)\right) \\
= & \left(\begin{array}{cc}
\frac{1}{a} \mathcal{F}_{p+1}(x) & \frac{d(x)}{a} \mathcal{F}_{p}(x) \\
\frac{1}{a} \mathcal{F}_{p}(x) & \frac{d(x)}{a} \mathcal{F}_{p-1}(x)
\end{array}\right) \cdot \sum_{j=0}^{n}\left(\begin{array}{c}
n \\
j
\end{array}\right) c^{j}(x) d^{n-j}(x)\left(\begin{array}{cc}
\frac{1}{a} \mathcal{F}_{j+1}(x) & \frac{d(x)}{a} \mathcal{F}_{j}(x) \\
\frac{1}{a} \mathcal{F}_{j}(x) & \frac{d(x)}{a} \mathcal{F}_{j-1}(x)
\end{array}\right) .
\end{aligned}
$$

Then by Corollary 2.7 and the $(1,2)$ entry of the first matrix in the above equality, we have

$$
\begin{aligned}
a \mathcal{F}_{2 n+p}(x) & =\sum_{j=0}^{n}\left(\begin{array}{l}
n \\
j
\end{array}\right) c^{j}(x) d^{n-j}(x)\left(\mathcal{F}_{p}(x) \mathcal{F}_{j+1}(x)+d(x) \mathcal{F}_{p-1}(x) \mathcal{F}_{j}(x)\right) \\
& =a \sum_{j=0}^{n}\left(\begin{array}{l}
n \\
j
\end{array}\right) c^{j}(x) d^{n-j}(x) \mathcal{F}_{j+p}(x) .
\end{aligned}
$$

Example 2.14 Let $\mathcal{F}_{n}(x)$ be the Fibonacci polynomial $F_{n}(x)$ in which $a=1, c(x)=x, d(x)=1$ in Eq. (1). By Theorem 2.13, we have

$$
F_{2 n+p}(x)=\sum_{j=0}^{n}\left(\begin{array}{l}
n \\
j
\end{array}\right) x^{j} F_{j+p}(x) .
$$

Given $n=2$ and $p=1$, we have

$$
F_{5}(x)=F_{1}(x)+2 x F_{2}(x)+x^{2} F_{3}(x) .
$$

Indeed, this equality holds for $F_{1}(x)=1, F_{2}(x)=x, F_{3}(x)=x^{2}+1$ and $F_{5}(x)=x^{4}+3 x^{2}+1$.

\section{Lucas Type Polynomials}

Based on the results of Fibonacci type $\mathcal{F}_{n}(x)$, some identities of Lucas type polynomials $\mathcal{L}_{n}(x)$ will be demonstrated in this section. Throughout this section, we assume $\mathcal{L}_{n}(x)$ and $\mathcal{F}_{n}(x)$ have the same recursive formula with $\mathcal{L}_{0}(x)=\mathcal{F}_{1}(x)$, that is, for $n \geq 2$,

$$
\mathcal{F}_{0}(x)=0, \mathcal{F}_{1}(x)=a \text { and } \mathcal{F}_{n}(x)=c(x) \mathcal{F}_{n-1}(x)+d(x) \mathcal{F}_{n-2}(x),
$$

and

$$
\mathcal{L}_{0}(x)=a, \mathcal{L}_{1}(x)=b(x) \text { and } \mathcal{L}_{n}(x)=c(x) \mathcal{L}_{n-1}(x)+d(x) \mathcal{L}_{n-2}(x)
$$

where $a \in \mathbb{R} \backslash\{0\}$. By applying Theorem 2.1, one can connect $\mathcal{L}_{n}(x)$ with $\mathcal{F}_{n}(x)$ below.

Theorem 3.1 Let $\mathcal{F}_{n}(x)$ and $\mathcal{L}_{n}(x)$ be the Fibonacci type polynomial and Lucas type polynomial respectively with $\mathcal{L}_{0}(x)=$ $\mathcal{F}_{1}(x)=a$. Then for each $n \in \mathbb{N}$,

$$
\left(\begin{array}{cc}
\mathcal{L}_{n+2}(x) & \mathcal{L}_{n+1}(x) \\
\mathcal{L}_{n+1}(x) & \mathcal{L}_{n}(x)
\end{array}\right)=\left(\begin{array}{cc}
\frac{b(x) c(x)+a d(x)}{a} & \frac{b(x) d(x)}{a} \\
\frac{b(x)}{a} & d
\end{array}\right)\left(\begin{array}{cc}
\mathcal{F}_{n+1}(x) & \mathcal{F}_{n}(x) \\
\mathcal{F}_{n}(x) & \mathcal{F}_{n-1}(x)
\end{array}\right)
$$

Proof. First, we will prove $\mathcal{L}_{n}(x)=\frac{b(x)}{a} \mathcal{F}_{n}(x)+d(x) \mathcal{F}_{n-1}(x)$ holds for each $n \in \mathbb{N}$. Let $n=1$. Then

$$
\mathcal{L}_{1}(x)=b(x)=\frac{b(x)}{a} \mathcal{F}_{1}(x)+d(x) \mathcal{F}_{0}(x)
$$

Let $n=2$. Then

$$
\mathcal{L}_{2}(x)=b(x) c(x)+a d(x)=\frac{b(x)}{a} \mathcal{F}_{2}(x)+d(x) \mathcal{F}_{1}(x)
$$


Assume this equality hods for $n=k-1$ and $k$. Let $n=k+1$. Then

$$
\begin{aligned}
\mathcal{L}_{k+1}(x) & =c(x) \mathcal{L}_{k}(x)+d(x) \mathcal{L}_{k-1}(x) \\
& =c(x)\left(\frac{b(x)}{a} \mathcal{F}_{k}(x)+d(x) \mathcal{F}_{k-1}(x)\right)+d(x)\left(\frac{b(x)}{a} \mathcal{F}_{k-1}(x)+d(x) \mathcal{F}_{k-2}(x)\right) \\
& =\frac{b(x)}{a}\left(c(x) \mathcal{F}_{k}(x)+d(x) \mathcal{F}_{k-1}(x)\right)+d(x)\left(c(x) \mathcal{F}_{k-1}(x)+d(x) \mathcal{F}_{k-2}(x)\right) \\
& =\frac{b(x)}{a} \mathcal{F}_{k+1}(x)+d(x) \mathcal{F}_{k}(x) .
\end{aligned}
$$

By induction, $\mathcal{L}_{n}(x)=\frac{b(x)}{a} \mathcal{F}_{n}(x)+d(x) \mathcal{F}_{n-1}(x)$ holds for all $n \in \mathbb{N}$. Also,

$$
\begin{aligned}
\mathcal{L}_{n}(x) & =\frac{b(x)}{a} \mathcal{F}_{n}(x)+d(x) \mathcal{F}_{n-1}(x) \\
& =\frac{b(x)}{a}\left(c(x) \mathcal{F}_{n-1}(x)+d(x) \mathcal{F}_{n-2}(x)\right)+d(x) \mathcal{F}_{n-1}(x) \\
& =\frac{b(x) c(x)+a d(x)}{a} \mathcal{F}_{n-1}(x)+\frac{b(x) d(x)}{a} \mathcal{F}_{n-2}(x) .
\end{aligned}
$$

One has the result by these two equalities

$$
\mathcal{L}_{n}(x)=\frac{b(x)}{a} \mathcal{F}_{n}(x)+d(x) \mathcal{F}_{n-1}(x)
$$

and

$$
\mathcal{L}_{n}(x)=\frac{b(x) c(x)+a d(x)}{a} \mathcal{F}_{n-1}(x)+\frac{b(x) d(x)}{a} \mathcal{F}_{n-2}(x) .
$$

Next, we will demonstrate the relation between Lucas type polynomials and the Fibonacci type $Q(x)$ matrix .

Theorem 3.2 Let $\mathcal{L}_{n}(x)$ be the Lucas type polynomial. Then for each $n \in \mathbb{N}$,

$$
\left(\begin{array}{cc}
\mathcal{L}_{n+2}(x) & d(x) \mathcal{L}_{n+1}(x) \\
\mathcal{L}_{n+1}(x) & d(x) \mathcal{L}_{n}(x)
\end{array}\right)=\left(\begin{array}{ll}
\mathcal{L}_{2}(x) & d(x) \mathcal{L}_{1}(x) \\
\mathcal{L}_{1}(x) & d(x) \mathcal{L}_{0}(x)
\end{array}\right) Q^{n}(x)
$$

Proof. By Theorem 2.1 and Theorem 3.1, we have

$$
\begin{aligned}
& \left(\begin{array}{cc}
\mathcal{L}_{n+2}(x) & d(x) \mathcal{L}_{n+1}(x) \\
\mathcal{L}_{n+1}(x) & d(x) \mathcal{L}_{n}(x)
\end{array}\right) \\
= & \left(\begin{array}{cc}
\mathcal{L}_{n+2}(x) & \mathcal{L}_{n+1}(x) \\
\mathcal{L}_{n+1}(x) & \mathcal{L}_{n}(x)
\end{array}\right)\left(\begin{array}{cc}
1 & 0 \\
0 & d(x)
\end{array}\right) \\
= & \left(\begin{array}{cc}
\frac{b(x) c(x)+a d(x)}{a} & \frac{b(x) d(x)}{a} \\
\frac{b(x)}{a} & d
\end{array}\right)\left(\begin{array}{cc}
\mathcal{F}_{n+1}(x) & \mathcal{F}_{n}(x) \\
\mathcal{F}_{n}(x) & \mathcal{F}_{n-1}(x)
\end{array}\right)\left(\begin{array}{cc}
1 & 0 \\
0 & d(x)
\end{array}\right) \\
= & \left(\begin{array}{cc}
b(x) c(x)+a d(x) & b(x) d(x) \\
b(x) & a d(x)
\end{array}\right)\left(\begin{array}{cc}
\frac{1}{a} \mathcal{F}_{n+1}(x) & \frac{d(x)}{a} \mathcal{F}_{n}(x) \\
\frac{1}{a} \mathcal{F}_{n}(x) & \frac{d(x)}{a} \mathcal{F}_{n-1}(x)
\end{array}\right) \\
= & \left(\begin{array}{cc}
\mathcal{L}_{2}(x) & d(x) \mathcal{L}_{1}(x) \\
\mathcal{L}_{1}(x) & d(x) \mathcal{L}_{0}(x)
\end{array}\right)\left(\begin{array}{cc}
c(x) & d(x) \\
1 & 0
\end{array}\right)^{n}
\end{aligned}
$$

for each $n \in \mathbb{N}$.

Using Theorem 3.2, one has the Cassini type identity for the Lucas type polynomial $\mathcal{L}_{n}(x)$.

Corollary 3.3 Let $\mathcal{L}_{n}(x)$ be the Lucas type polynomial. Then for each $n \in \mathbb{N}$,

$$
\mathcal{L}_{n+2}(x) \mathcal{L}_{n}(x)-\mathcal{L}_{n+1}^{2}(x)=\left(\mathcal{L}_{2}(x) \mathcal{L}_{0}(x)-\mathcal{L}_{1}^{2}(x)\right)(-d(x))^{n} .
$$


Proof. By Theorem 3.2, we have

$$
\operatorname{det}\left(\begin{array}{ll}
\mathcal{L}_{n+2}(x) & d(x) \mathcal{L}_{n+1}(x) \\
\mathcal{L}_{n+1}(x) & d(x) \mathcal{L}_{n}(x)
\end{array}\right)=\operatorname{det}\left(\begin{array}{ll}
\mathcal{L}_{2}(x) & d(x) \mathcal{L}_{1}(x) \\
\mathcal{L}_{1}(x) & d(x) \mathcal{L}_{0}(x)
\end{array}\right)\left(\operatorname{det}\left(\begin{array}{cc}
c(x) & d(x) \\
1 & 0
\end{array}\right)\right)^{n} .
$$

Hence

$$
\mathcal{L}_{n+2}(x) \mathcal{L}_{n}(x)-\mathcal{L}_{n+1}^{2}(x)=\left(\mathcal{L}_{2}(x) \mathcal{L}_{0}(x)-\mathcal{L}_{1}^{2}(x)\right)(-d(x))^{n}
$$

Example 3.4 Let $a=2, b(x)=2 x, c(x)=2 x, d(x)=1$ in Eq. (2). Then $\mathcal{L}_{n}(x)=D_{n}(x)$ is the Pell-Lucas polynomial as defined in Table 1. By Corollary 3.3, the Cassini identity for the Pell-Lucas polynomial $D_{n}(x)$ is

$$
D_{n+2}(x) D_{n}(x)-D_{n+1}^{2}(x)=\left(4 x^{2}+4\right)(-1)^{n} .
$$

By Corollary 3.3, we have the result below.

Corollary 3.5 Let $\mathcal{L}_{n}(x)$ be the Lucas type polynomial. Then for each $n \in \mathbb{N}$,

$$
c(x) \mathcal{L}_{n+1}(x) \mathcal{L}_{n}(x)+d(x) \mathcal{L}_{n}^{2}(x)-\mathcal{L}_{n+1}^{2}(x)=\left(\mathcal{L}_{2}(x) \mathcal{L}_{0}(x)-\mathcal{L}_{1}^{2}(x)\right)(-d(x))^{n} .
$$

Proof. By

$$
\mathcal{L}_{n+2}(x) \mathcal{L}_{n}(x)-\mathcal{L}_{n+1}^{2}(x)=\left(\mathcal{L}_{2}(x) \mathcal{L}_{0}(x)-\mathcal{L}_{1}^{2}(x)\right)(-d(x))^{n}
$$

and

$$
\mathcal{L}_{n+2}(x)=c(x) \mathcal{L}_{n+1}(x)+d(x) \mathcal{L}_{n}(x),
$$

we have

$$
\begin{aligned}
& \left(\mathcal{L}_{2}(x) \mathcal{L}_{0}(x)-\mathcal{L}_{1}^{2}(x)\right)(-d(x))^{n} \\
= & \left(c(x) \mathcal{L}_{n+1}(x)+d(x) \mathcal{L}_{n}(x)\right) \mathcal{L}_{n}(x)-\mathcal{L}_{n+1}^{2}(x) \\
= & c(x) \mathcal{L}_{n+1}(x) \mathcal{L}_{n}(x)+d(x) \mathcal{L}_{n}^{2}(x)-\mathcal{L}_{n+1}^{2}(x) .
\end{aligned}
$$

Using $Q^{2}(x)=c(x) Q(x)+d(x) I$ again, we have the expression of $\mathcal{L}_{n}(x)$.

Theorem 3.6 Let $\mathcal{L}_{n}(x)$ be the Lucas type polynomial. Then for each $n, p \in \mathbb{N}$,

$$
\mathcal{L}_{2 n+p}(x)=\sum_{j=0}^{n}\left(\begin{array}{l}
n \\
j
\end{array}\right) c^{j}(x) d^{n-j}(x) \mathcal{L}_{p+j}(x) .
$$

Proof. By Theorem 3.2, we have

$$
\begin{aligned}
& \left(\begin{array}{ll}
\mathcal{L}_{2 n+p+2}(x) & d(x) \mathcal{L}_{2 n+p+1}(x) \\
\mathcal{L}_{2 n+p+1}(x) & d(x) \mathcal{L}_{2 n+p}(x)
\end{array}\right) \\
& =\left(\begin{array}{ll}
\mathcal{L}_{2}(x) & d(x) \mathcal{L}_{1}(x) \\
\mathcal{L}_{1}(x) & d(x) \mathcal{L}_{0}(x)
\end{array}\right) Q^{2 n+p}(x) \\
& =\left(\begin{array}{ll}
\mathcal{L}_{2}(x) & d(x) \mathcal{L}_{1}(x) \\
\mathcal{L}_{1}(x) & d(x) \mathcal{L}_{0}(x)
\end{array}\right) Q^{p}(x)\left(Q^{2}(x)\right)^{n} \\
& =\left(\begin{array}{cc}
\mathcal{L}_{p+2}(x) & d(x) \mathcal{L}_{p+1}(x) \\
\mathcal{L}_{p+1}(x) & d(x) \mathcal{L}_{p}(x)
\end{array}\right)(c(x) Q(x)+d(x) I)^{n} \\
& =\left(\begin{array}{cc}
\mathcal{L}_{p+2}(x) & d(x) \mathcal{L}_{p+1}(x) \\
\mathcal{L}_{p+1}(x) & d(x) \mathcal{L}_{p}(x)
\end{array}\right)\left(\sum_{j=0}^{n}\left(\begin{array}{c}
n \\
j
\end{array}\right) c^{j}(x) d^{n-j}(x) Q^{j}(x)\right) \\
& =\sum_{j=0}^{n}\left(\begin{array}{l}
n \\
j
\end{array}\right) c^{j}(x) d^{n-j}(x)\left(\begin{array}{cc}
\mathcal{L}_{p+j+2}(x) & d(x) \mathcal{L}_{p+j+1}(x) \\
\mathcal{L}_{p+j+1}(x) & d(x) \mathcal{L}_{p+j}(x)
\end{array}\right)
\end{aligned}
$$


By considering the $(2,2)$ entry of the first matrix in the above equality, we have

$$
\mathcal{L}_{2 n+p}(x)=\sum_{j=0}^{n}\left(\begin{array}{l}
n \\
j
\end{array}\right) c^{j}(x) d^{n-j}(x) \mathcal{L}_{p+j}(x) .
$$

Example 3.7 Let $\mathcal{L}_{n}(x)$ be the Morgan-Voyce polynomial $C_{n}(x)$ in which $a=2, b(x)=x+2, c(x)=x+2, d(x)=-1$ in Eq. (2). By Theorem 3.6, we have

$$
C_{2 n+p}(x)=\sum_{j=0}^{n}\left(\begin{array}{l}
n \\
j
\end{array}\right)(x+2)^{j}(-1)^{n-j} C_{p+j}(x) .
$$

Finally, we end up this note by providing two identities in which $\mathcal{F}_{n}(x)$ and $\mathcal{L}_{n}(x)$ are involved.

Proposition 3.8 Let $\mathcal{F}_{n}(x)$ and $\mathcal{L}_{n}(x)$ be the Fibonacci type polynomial and Lucas type polynomial respectively with $\mathcal{L}_{0}(x)=\mathcal{F}_{1}(x)=a$. Then for each $n, m \in \mathbb{N}$,

$$
a \mathcal{L}_{n+m}(x)=\mathcal{L}_{n+1}(x) \mathcal{F}_{m}(x)+d(x) \mathcal{L}_{n}(x) \mathcal{F}_{m-1}(x) .
$$

Proof. By Theorem 3.2, we have

$$
\begin{aligned}
& \left(\begin{array}{ll}
\mathcal{L}_{n+m+2}(x) & d(x) \mathcal{L}_{n+m+1}(x) \\
\mathcal{L}_{n+m+1}(x) & d(x) \mathcal{L}_{n+m}(x)
\end{array}\right) \\
= & \left(\begin{array}{ll}
\mathcal{L}_{2}(x) & d(x) \mathcal{L}_{1}(x) \\
\mathcal{L}_{1}(x) & d(x) \mathcal{L}_{0}(x)
\end{array}\right) Q^{n}(x) Q^{m}(x) \\
= & \left(\begin{array}{cc}
\mathcal{L}_{n+2}(x) & d(x) \mathcal{L}_{n+1}(x) \\
\mathcal{L}_{n+1}(x) & d(x) \mathcal{L}_{n}(x)
\end{array}\right)\left(\begin{array}{cc}
\frac{1}{a} \mathcal{F}_{m+1}(x) & \frac{d(x)}{a} \mathcal{F}_{m}(x) \\
\frac{1}{a} \mathcal{F}_{m}(x) & \frac{d(x)}{a} \mathcal{F}_{m-1}(x)
\end{array}\right) .
\end{aligned}
$$

Then by the $(2,2)$ entry of the first matrix in the above equality, we have

$$
a \mathcal{L}_{n+m}(x)=\mathcal{L}_{n+1}(x) \mathcal{F}_{m}(x)+d(x) \mathcal{L}_{n}(x) \mathcal{F}_{m-1}(x)
$$

for each $n, m \in \mathbb{N}$.

Example 3.9 Let $\mathcal{F}_{n}(x)$ and $\mathcal{L}_{n}(x)$ be the Jacobsthal polynomial $J_{n}(x)$ and the Jacobsthal-Lucas polynomial $\Lambda_{n}(x)$ respectively, as defined in Table 1. Then $\Lambda_{0}(x)=J_{1}(x)=1$ which satisfies the condition in Proposition 3.8. Hence we have the following equality for $J_{n}(x)$ and $\Lambda_{n}(x)$ :

$$
\Lambda_{n+m}(x)=\Lambda_{n+1}(x) J_{m}(x)+2 x \Lambda_{n}(x) J_{m-1}(x) .
$$

Proposition 3.10 Let $\mathcal{F}_{n}(x)$ and $\mathcal{L}_{n}(x)$ be the Fibonacci type polynomial and Lucas type polynomial respectively with $\mathcal{L}_{0}(x)=\mathcal{F}_{1}(x)=a$. Let $d(x) \neq 0$ for each $x \in \mathbb{R}$. Then for each $n, m \in \mathbb{N}$ with $n \geq m$,

$$
a(-d(x))^{m} \mathcal{L}_{n-m}(x)=\mathcal{L}_{n}(x) \mathcal{F}_{m+1}(x)-\mathcal{L}_{n+1}(x) \mathcal{F}_{m}(x) .
$$

Proof. By Theorem 3.2 and $Q^{n-m}(x)=Q^{n}(x) Q^{-m}(x)$, we have

$$
\begin{aligned}
& \left(\begin{array}{ll}
\mathcal{L}_{n-m+2}(x) & d(x) \mathcal{L}_{n-m+1}(x) \\
\mathcal{L}_{n-m+1}(x) & d(x) \mathcal{L}_{n-m}(x)
\end{array}\right) \\
= & \left(\begin{array}{cc}
\mathcal{L}_{2}(x) & d(x) \mathcal{L}_{1}(x) \\
\mathcal{L}_{1}(x) & d(x) \mathcal{L}_{0}(x)
\end{array}\right) Q^{n}(x) Q^{-m}(x) \\
= & \left(\begin{array}{cc}
\mathcal{L}_{n+2}(x) & d(x) \mathcal{L}_{n+1}(x) \\
\mathcal{L}_{n+1}(x) & d(x) \mathcal{L}_{n}(x)
\end{array}\right) \frac{1}{(-d(x))^{m}}\left(\begin{array}{cc}
\frac{d(x)}{a} \mathcal{F}_{m-1}(x) & -\frac{d(x)}{a} \mathcal{F}_{m}(x) \\
-\frac{1}{a} \mathcal{F}_{m}(x) & \frac{1}{a} \mathcal{F}_{m+1}(x)
\end{array}\right) .
\end{aligned}
$$


Then considering the $(2,2)$ entry of the first matrix in the above equality, we have

$$
a(-d(x))^{m} \mathcal{L}_{n-m}(x)=\mathcal{L}_{n}(x) \mathcal{F}_{m+1}(x)-\mathcal{L}_{n+1}(x) \mathcal{F}_{m}(x) .
$$

Example 3.11 Let $\mathcal{F}_{n}(x)$ and $\mathcal{L}_{n}(x)$ be the Jacobsthal polynomial $J_{n}(x)$ and the Jacobsthal-Lucas polynomial $\Lambda_{n}(x)$ respectively. Then $\Lambda_{0}(x)=J_{1}(x)=1$ and

$$
(-2 x)^{m} \Lambda_{n-m}(x)=\Lambda_{n}(x) J_{m+1}(x)-\Lambda_{n+1}(x) J_{m}(x) .
$$

\section{Acknowledgements}

The authors were supported by grant MOST 109-2115-M-142-002- of Ministry of Science and Technology, Taiwan.

\section{References}

André-Jeannin, R. (1994). A note on a general class of polynomials. Fibonacci Quarterly, 32, 445-454.

André-Jeannin, R. (1995). A note on a general class of polynomials II. Fibonacci Quarterly, 33, 341-351.

Falcón, S., \& Plaza, Á. (2007). On the Fibonacci k-numbers. Chaos, Solitons and Fractals, 32, 1615-1624. https://doi.org/10.1016/j.chaos.2006.09.022

Falcón, S., \& Plaza, Á. (2009). On $k$-Fibonacci sequences and polynomials and their derivatives. Chaos, Solitons and Fractals, 39, 1005-1009. https://doi.org/10.1016/j.chaos.2007.03.007

Flórez, R., Higuita, R., \& Mukherjee, A. (2018). Characterization of the strong divisibility property for generalized Fibonacci polynomials. Intergers, 18, Paper No. A14.

Flórez, R., McAnally, N., \& Mukherjee, A. (2018). Identities for the generalized Fibonacci polynomial. Intergers, 18B, Paper No. A2.

Gould, H. W. (1981). A history of Fibonacci $Q$-matrix and a higher-dimensional problem. Fibonacci Quarterly, 19, 250-257.

Horadam, A. F. (1979). Chebyshev and Fermat polynomials for diagonal functions. Fibonacci Quarterly, 17, 328-333.

Horadam, A. F., \& Mahon, J. M. (1985). Pell and Pell-Lucas polynomials. Fibonacci Quarterly, 23, 7-20.

Koshy, T. (2001). Fibonacci and Lucas numbers with applications. New York, John Wiley. https://doi.org/10.1002/9781118033067

Lin, Y-J. (2012). The generalization and application of Fibonacci $Q$-matrix on recursive sequences. Mathmedia (Chinese edition), 36, 52-63. https://doi.org/10.2307/2790741

Wu, Z., \& Zhang, W. (2013). Several identities involving the Fibonacci polynomials and Lucas polynomials. J. Inequal. Appl., 2013, Paper No. 205. https://doi.org/10.1186/1029-242X-2013-205

\section{Copyrights}

Copyright for this article is retained by the author(s), with first publication rights granted to the journal.

This is an open-access article distributed under the terms and conditions of the Creative Commons Attribution license (http://creativecommons.org/licenses/by/4.0/). 